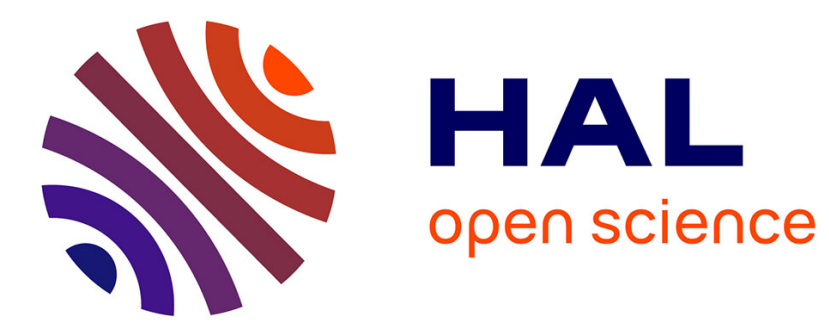

\title{
Motion Planning and Irreducible Trajectories
}

\author{
Andreas Orthey, Olivier Stasse, Florent Lamiraux
}

\section{To cite this version:}

Andreas Orthey, Olivier Stasse, Florent Lamiraux. Motion Planning and Irreducible Trajectories.

ICRA, May 2015, Seattle, United States. hal-01121025

\section{HAL Id: hal-01121025 \\ https://hal.science/hal-01121025}

Submitted on 2 Mar 2015

HAL is a multi-disciplinary open access archive for the deposit and dissemination of scientific research documents, whether they are published or not. The documents may come from teaching and research institutions in France or abroad, or from public or private research centers.
L'archive ouverte pluridisciplinaire HAL, est destinée au dépôt et à la diffusion de documents scientifiques de niveau recherche, publiés ou non, émanant des établissements d'enseignement et de recherche français ou étrangers, des laboratoires publics ou privés. 


\title{
Motion Planning and Irreducible Trajectories
}

\author{
Andreas Orthey ${ }^{1}$, Olivier Stasse ${ }^{1}$, Florent Lamiraux ${ }^{1}$
}

\begin{abstract}
We introduce a novel notion for lowering the dimensionality of motion planning problems: Irreducibility. Irreducibility of a configuration space trajectory $\tau$ means: We cannot find another configuration space trajectory $\tau^{\prime}$, such that the swept volume of $\tau^{\prime}$ is included in the swept volume of $\tau$. The main contribution of our work is twofold: First, we show that motion planning in the space of irreducible trajectories is complete. Second, we show that we can construct reducible subspaces by reasoning about the inherent hierarchical structure of open kinematic chains. Using those theoretical results, we proceed by analytically defining a 7 -dimensional irreducible configuration subspace for the humanoid robot HRP-2 under some assumptions. To show its practical importance, we solve a high-dimensional pin-hole problem for HRP-2 from the scratch.
\end{abstract}

\section{INTRODUCTION}

Generating movements for arbitrary mechanical system is a first basic requirement for higher cognitive functions. Motion planning is the systematic study of how we can generate movements from an algorithmic perspective. While successful applications have already emerged, we know that general motion planning is NP-hard [1]. Complex scenarios with humanoid robots require extensive computational time, where solutions require the prespecification of waypoints, and even then require for instance 1 hour to crouch through a tunnel [2] or 2 hours to walk through a wall [3]. This prespecification and long computational time are a severe restriction for reactive movements, and constitute therefore a bottleneck for the longterm-goal of understanding intelligent behavior. Our goal in this paper is to show directions of how we can possibly overcome this bottleneck.

As a starting point, let us point out that the high computational time of motion planning is mainly caused by the dimensionality of the configuration space. Large parts of the configuration space are searched, even though they do not increase our knowledge about the feasibility of the problem at hand. From our perspective, it is clear that we need to precompute the parts of the configuration space which are important and those which are not important. Such a precomputation will be called a motion prior.

An important motion prior can be obtained by analyzing the mechanical system of the planning problem. In particular, we want to show that the prior knowledge of the mechanical system can reduce the configuration space dimensionality. Our contribution is to 1) introduce the concept of an irreducible trajectory, which states that there is no other

\footnotetext{
* This research has received funding from the European Union Seventh Framework Programme (FP7/2007 - 2013) under grant agreement $n^{\circ}$ 611909, KoroiBot.

1 are with the CNRS, LAAS, 7 av. du Colonel Roche, F-31400, Toulouse, France, Univ de Toulouse, LAAS, F-31400, Toulouse, France \{aorthey, ostasse, flamiraux\} at laas.fr
}

trajectory with a smaller swept volume. 2) We show that planning in the space of irreducible trajectories is complete. 3) We show a practical, but non-complete way to construct the space of irreducible trajectories by using the cross section of the workspace volume of configurations. 4) We show how one can take advantage of the hierarchical decomposition of the kinematic chain of a robot to create reducible subspaces, 5) we use our theoretical results to define an irreducible configuration subspace for the humanoid robot HRP-2, and 6) we use this irreducible configuration subspace to plan a feasible motion for HRP-2 in a highly restricted environment, where the solution trajectory needs to traverse a pin-hole in the configuration space.

\section{RELATED WORK}

Humanoid motion planning is important for industrial applications and likewise for further understanding the algorithmic study of how to generate motions. Research for the general problem in arbitrary environments has focused on multi-contact planning [2], with applications for example for climbing robots [4].

Our approach tries to establish a link between workspace and configuration space, similar to [5], but with the main idea that we can reduce the planning dimensionality by precomputation. Precomputation in motion planning can be classified into three categories: Robot, Environment and Behavior. Most ideas have concentrated on both robot and environment simultaneously, either for a single or multiple environments.

Precomputation in a single environment was addressed by Manocha et al. [6], who learned a mapping between configuration space and feasiblity, and by Likhachev et al. [7] who precomputed RRT nodes, storing the graph structure for later reuse. Both approaches need time to learn the mapping, but work well once the environment is sufficiently sampled. Complementary to this, we projected the configuration space onto a lower dimensional subspace, such that both approaches can be used without modifications.

In multiple environments, Jetchev et al. [8] learned a mapping from environment to configuration space trajectories. Hauser et al. [9] stored configuration space trajectories and used a similiarity metric to reuse them in a new environment. And Berenson et al. [10] store configuration space trajectories and use the distance of start and goal configuration for choosing a stored trajectory in a new environment. Again, our approach could be used here to reduce the database of motions by storing only the nodes in the irreducible subspace. 


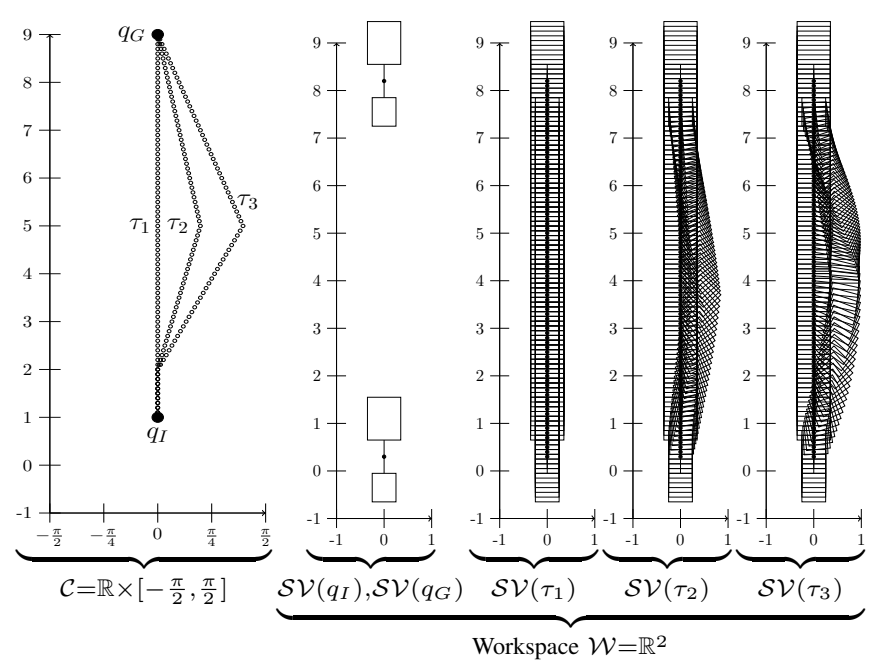

Fig. 1: Explanatory example of irreducible trajectories for a 2-link, 2-dof robot, which can move along the $y$-axis, and which has one rotational joint between its two links, such that its configuration space is $\mathcal{C}=$ $\mathbb{R} \times\left[-\frac{\pi}{2}, \frac{\pi}{2}\right]$. Left. Three configuration space trajectories $\tau_{1}, \tau_{2}, \tau_{3}$ with $\tau_{1}(0)=\tau_{2}(0)=\tau_{3}(0)=q_{I}, \tau_{1}(1)=\tau_{2}(1)=\tau_{3}(1)=q_{G}$. Right. The workspace volume of the starting configurations $q_{I}, q_{G}$, and the swept volume of the three trajectories, whereby we have that $\mathcal{S} \mathcal{V}\left(\tau_{1}\right) \subset \mathcal{S} \mathcal{V}\left(\tau_{2}\right)$ and $\mathcal{S} \mathcal{V}\left(\tau_{1}\right) \subset \mathcal{S} \mathcal{V}\left(\tau_{3}\right)$, i.e. $\tau_{2}$ and $\tau_{3}$ are reducible by $\tau_{1}$, and $\tau_{1}$ is in fact irreducible.

Finally, if the robot, the environment and the behavior are known, attractor based models can be learned for efficiently storing motions, examples are funnel databases [11] and dynamical motion primitives (DMP) [12].

\section{Motion Planning PREliminaries}

We restate relevant motion planning definitions, following the classical formulation by [13, Chapter 4]

Definition 1 (Motion Planning Problem). Let $A=$ $\left\{q_{I}, q_{G}, \mathcal{R}, \mathbf{E}\right\}$ be a motion planning problem, with $\mathcal{R}$ the robotic system, $q_{I}$ the initial configuration, $q_{G}$ the goal configuration, and $\mathbf{E}$ the environment.

Definition 2 (Configuration Space Trajectory). Let $A$ be given. Then we denote by $\mathcal{F}\left(q_{I}, q_{G}\right)=C^{1}([0,1], \mathcal{C})$ the set of continuously differentiable functions from $[0,1]$ to the configuration space $\mathcal{C}$, with the property that if $\tau \in$ $\mathcal{F}\left(q_{I}, q_{G}\right) \Rightarrow \tau(0)=q_{I}, \tau(1)=q_{G}$.

Definition 3 (Swept Volume). The workspace volume swept by the trajectory $\tau \in \mathcal{F}\left(q_{I}, q_{G}\right)$ will be denoted by $\mathcal{S} \mathcal{V}(\tau)$.

Definition 4 (Feasible Trajectory). A trajectory $\tau \in$ $\mathcal{F}\left(q_{I}, q_{G}\right)$ is called feasible in an environment $\mathbf{E}$, if $\mathcal{S} \mathcal{V}(\tau) \cap$ $\mathbf{E}=\emptyset$.

Definition 5 (Feasible Configuration Space Trajectory). Let $\mathcal{S} \subset \mathcal{F}\left(q_{I}, q_{G}\right)$ be a set of Configuration space trajectories. Let $A$ be a specific motion planning problem. If there exist $\tau \in \mathcal{S}$ such that $\tau$ solves $A$, then $\mathcal{S}$ is said to be feasible w.r.t. A.

We will not consider here physical feasibility of the robot, like joint limits or self collision. We will postpone this discussion until Section VII.

\section{IRREDUCIBLE TRAJECTORIES}

We denote by $\subset$ the proper subset. Let $A=\left\{q_{I}, q_{G}, \mathcal{R}, \mathbf{E}\right\}$ be given, and let $\mathcal{F}=\mathcal{F}\left(q_{I}, q_{G}\right)$.

Definition 6. A trajectory $\tau^{\prime} \in \mathcal{F}$ is called reducible, if there exist $\tau \in \mathcal{F}$ such that $\mathcal{S} \mathcal{V}(\tau) \subset \mathcal{S} \mathcal{V}\left(\tau^{\prime}\right)$. Otherwise $\tau^{\prime}$ is called irreducible.

Fig. 1 provides a visualization of the irreducible definition for trajectories. One can see three configuration space trajectories $\tau_{1}, \tau_{2}, \tau_{3}$, and its swept volumes in workspace. Applying the definition, we have that $\tau_{2}$ and $\tau_{3}$ are reducible by $\tau_{1}$. We will now show why irreducibility is important for motion planning.

Theorem 1. Let $\tau, \tau^{\prime} \in \mathcal{F}$ be such that $\mathcal{S} \mathcal{V}(\tau) \subset \mathcal{S V}\left(\tau^{\prime}\right)$, i.e. $\tau^{\prime}$ is reduced by $\tau$.

$$
\begin{gathered}
\text { If } \tau \text { is infeasible } \Rightarrow \tau^{\prime} \text { is infeasible } \\
\text { If } \tau^{\prime} \text { is feasible } \Rightarrow \tau \text { is feasible }
\end{gathered}
$$

Proof in Appendix.

Definition 7 (Irreducible Trajectories). Let the set of all irreducible configuration space trajectories be defined as

$$
\mathcal{I}=\{\tau \in \mathcal{F} \mid \tau \text { is irreducible }\}
$$

Lemma 1. Let $\tau \in \mathcal{F} \backslash \mathcal{I}$. Then there exist $\tau^{\prime} \in \mathcal{I}$, with $\mathcal{S} \mathcal{V}\left(\tau^{\prime}\right) \subset \mathcal{S} \mathcal{V}(\tau)$

Proof in Appendix.

Theorem 2. If $\mathcal{I}$ is infeasible $\Rightarrow \mathcal{F}$ is infeasible

Proof in Appendix.

\section{Corollary 1. Motion planning is complete in $\mathcal{I}$}

\section{Proof in Appendix.}

Going back to the example in Fig. 1, we can now make the statement, that trajectories $\tau_{2}$ and $\tau_{3}$ can be ignored for motion planning, while still being complete.

\section{IRREDUCIBLE CONFIGURATIONS}

In the preceding section, we showed completeness in the irreducible trajectory space. However, we haven't provided an exact practical method to construct this space. To develop a construction method, we approximate the swept volume by its cross section onto the movement direction. The next sections will study the irreducible property for those cross sections, i.e. how to formally define the irreducible configuration space based on cross sections, and how the knowledge of hierarchies of kinematic chains can provide a tool to practically construct a subspace.

Definition 8 (Cross Section Operator). Let the workspace volume of a configuration $q \in \mathcal{C}$ be $\mathcal{S V}(q)$. Let us assume w.l.o.g. that the robot is moving into the $y$-axis direction of the workspace. Let $Y=\left\{\left(x_{0}, x_{1}, x_{2}\right)^{T} \in \mathbb{R}^{3} \mid x_{1}=0\right\}$ be the $x$-z plane. We define the cross section operator $V(q)$ as the orthogonal projection of $\mathcal{S V}(q)$ onto the $x-z$ plane: 


$$
V: \mathcal{C} \rightarrow Y
$$

Since computing the complete swept volume for a robot involves computationally expensive operations [14], we do the following assumption: The swept volume of a robot is approximated by its cross section operator, i.e. $q \in \mathcal{C}$ : $\mathcal{S} \mathcal{V}(q) \approx V(q)$, i.e. the swept volume is collapsed onto its cross section in movement direction.

Definition 9 (Reducible Configuration). A configuration $q^{\prime} \in$ $\mathcal{C}$ is called reducible, if there exist $q \in \mathcal{C}$, such that $V(q) \subset$ $V\left(q^{\prime}\right)$. Otherwise, $q^{\prime}$ is called irreducible.

Definition 10 (Feasible Configuration). A configuration $q \in$ $\mathcal{C}$ is called feasible in an environment $\mathbf{E}$, if for all $v \in V(q)$, we have $v \cap \mathbf{E}=\emptyset$.

Theorem 3. Let $q^{\prime} \in \mathcal{C}$ be reducible by $q \in \mathcal{C}$. Then we have

If $q$ is infeasible $\Rightarrow q^{\prime}$ is infeasible

If $q^{\prime}$ is feasible $\Rightarrow q$ is feasible

Proof in Appendix.

Definition 11 (Irreducible Configuration Set). We define the set of irreducible configurations as

$$
\begin{aligned}
Q & =\{q \in \mathcal{C} \mid q \text { is irreducible }\} \\
& =\left\{q \in \mathcal{C} \mid \neg \exists q^{\prime} \in \mathcal{C}: V\left(q^{\prime}\right) \subset V(q)\right\}
\end{aligned}
$$

with $\subset$ denoting the proper subset.

Theorem 4. Let $Q \subset \mathcal{C}$ be the irreducible set of $\mathcal{C}$. Then we have:

$$
\text { If } Q \text { is infeasible } \Rightarrow \mathcal{C} \text { is infeasible }
$$

\section{Proof in Appendix.}

As a note, Theorem 4 is valid only, because we defined feasibility by its cross section volume. Therefore, in general we cannot guarantee that motion planning is complete for irreducible configurations.

\section{Hierarchical IRREDUCIBILITy TheOREMS}

This section studies how the hierarchy of kinematic chains can be used to construct $Q$ analytically. It requires us to restrict our analysis to robotic systems, which can be represented by an open kinematic chain.

Assumption 1. $\mathcal{R}$ is representable by an open kinematic chain.

Lemma 2 (Translation Invariance). Let $q, p \in \mathcal{C}$, and $\mathbf{T}$ being the translation operator.

$$
V(p) \subset V(q) \Leftrightarrow \mathbf{T}(V(p)) \subset \mathbf{T}(V(q))
$$

Proof in Appendix.

Lemma 3 (Movement Direction Invariance). Let $q, p \in \mathcal{C}$, and $\mathbf{R}_{\mathrm{Y}}$ being the rotation around the $y$ axis.

$$
V(p) \subset V(q) \Leftrightarrow \mathbf{R}_{\mathrm{Y}}(V(p)) \subset \mathbf{R}_{\mathrm{Y}}(V(q))
$$

Proof in Appendix.

Definition 12 (Joint $J_{j}$ ). Let $J_{j}$ be a joint in the kinematic chain of $\mathcal{R}, x_{j} \in \mathbb{R}^{6}$ the position of joint $J_{j}$ in the world frame, and $H_{j}$ the transformation from the world frame to $J_{j}$.

Definition 13. Let $\mathcal{C}_{p}\left(J_{j}\right)$ be the subspace of parent configurations of $J_{j}$, including joint $J_{j}$. Let $\mathcal{C}_{c}\left(J_{j}\right)$ be the subspace of configurations of child joints of $J_{j}$, excluding joint $J_{j}$, i.e. such that we have a decomposition $\mathcal{C}=\mathcal{C}_{p} \cup \mathcal{C}_{c}$.

Let us assume w.l.o.g. that the rotational component of $H_{j}$, named $R\left(H_{j}\right)$, is equal to the rotational component of the world coordinate frame o, i.e. the transformation is in fact a translation $H_{j}=T_{j}$. Meaning, that $V$ projects onto the $x-z$ plane, as desired.

Definition 14. Let $x_{j}$ denote a specific workspace position of the joint $J_{j}$. Let $\mathbf{T}$ be the xyz translation operator, and $\mathbf{R}_{\mathrm{Y}}$ the rotational operator around the y-axis. Let us define the set of inverse kinematic configurations of $x_{j}$ as

$$
\begin{aligned}
P\left(x_{j}, J_{j}\right) & =\left\{q \in \mathcal{C}_{p}\left(J_{j}\right) \mid H_{j}(q)=x_{j}\right\} \\
P_{\mathbf{T}}\left(x_{j}, J_{j}\right) & =\left\{q \in \mathcal{C}_{p}\left(J_{j}\right) \mid H_{j}(q)=\mathbf{T}\left(x_{j}\right)\right\} \\
P_{\mathbf{R}_{\mathbf{Y}}}\left(x_{j}, J_{j}\right) & =\left\{q \in \mathcal{C}_{p}\left(J_{j}\right) \mid H_{j}(q)=\mathbf{R}_{\mathbf{Y}}\left(x_{j}\right)\right\}
\end{aligned}
$$

Theorem 5. Let $x_{j}, J_{j}$ be given. $\forall p \in P\left(x_{j}, J_{j}\right)$ :

$$
\begin{array}{r}
\text { if } \exists c^{\prime} \in \mathcal{C}_{c}\left(J_{j}\right) \text { with } V\left(c^{\prime}\right) \subset V(p) \\
\Rightarrow \forall c \in \mathcal{C}_{c}\left(N_{j}\right): V\left(c^{\prime} \cup p\right) \subset V(c \cup p) \text {, i.e. }
\end{array}
$$

$$
c \cup p \text { is reducible by } c^{\prime} \cup p
$$

Proof in Appendix.

Theorem 6. Theorem 5 holds $\forall p \in P_{\mathbf{T}}\left(x_{j}, J_{j}\right)$

Proof in Appendix.

Theorem 7. Theorem 5 holds $\forall p \in P_{\mathbf{R}_{\mathbf{Y}}}\left(x_{j}, J_{j}\right)$

Proof in Appendix.

We will illustrate Theorems 5,6 and 7 by a simple robot in the plane.

Example 1. Let $\mathcal{R}=\left\{\left\{L_{1}, L_{2}, L_{3}\right\},\left\{J_{0}, J_{1}, J_{2}\right\}\right\}$, with $J_{0}$ the free flyer of $L_{1}$, and choose $J_{0}$ as the root joint. Then $\mathcal{C}_{p}\left(J_{0}\right)=\left\{x \in \mathbb{R}^{2}, \theta \in[-\pi, \pi]\right\}$ and $\mathcal{C}_{c}\left(J_{0}\right)=\left\{q_{1}, q_{2} \in\right.$ $[-\pi, \pi]\}$. The robot is shown in Fig. 2 in configuration $q=$ $\left\{q_{p}, q_{c}\right\}$ with $q_{p}=(0,1,0)$ and $q_{c}=(0,0)$. Theorem 5 states that configuration $q_{c}$ of $\mathcal{C}_{c}$ reduces all other configurations of $\mathcal{C}_{c}$, since $V\left(q_{c}\right) \subset V\left(q_{p}\right)$. Theorem 6 states that we can translate the workspace structure arbitrarily without changing the inclusion property. And Theorem 7 states that we can rotate the workspace structure around the $x_{1}$-axis without changing the inclusion property. 


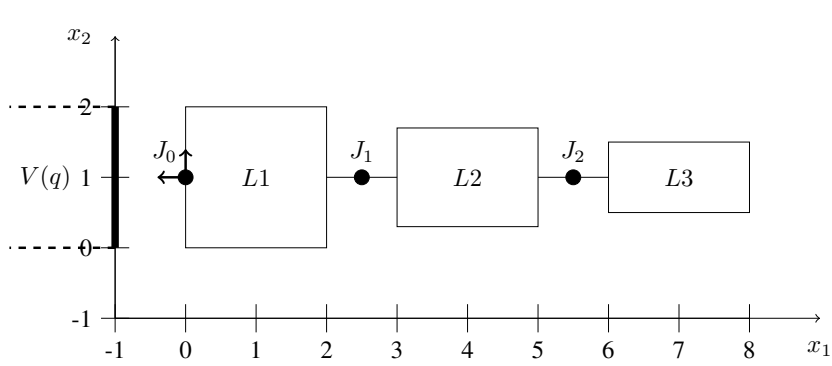

Fig. 2: 2-dof robot with free flyer in configuration $q=\left(x_{1}=1, x_{2}=\right.$ $\left.1, \theta=0, q_{1}=0, q_{2}=0\right)$. The cross section volume is given by $V(q)$, and $q_{1}, q_{2}$ are chosen such that Theorem 5 applies, meaning that $q_{1}, q_{2}$ are reducible.

\section{CONSTRAINT MANIFOLD}

Irreducibility provides a possible way to lower the dimensionality of the motion planning problem. However, even in the space of irreducible configurations, we have configurations which are physically feasible and ones who are not. Let us define the physical constraints in our case:

Definition 15 (Forward movement constraint manifold). Let $v$ be the movement direction of $\operatorname{COM}(q)$. A configuration $q \in \mathcal{C}$ belongs to the forward movement constraint manifold $\mathcal{C}_{F M}$, if it is (1) physically feasible, i.e. the following constraints are fulfilled:

- $q_{L} \leq q \leq q_{U}$ (Joint Limits)

- $\forall q_{i}, q_{j} \in q: d\left(q_{i}, q_{j}\right)>0, i \neq j$ (Self-Collision)

- $\operatorname{COM}(q) \in$ SupportPolygon (q) (Static Stability)

and (2) the robot can be moved along $v$ with infinitesimal small movements without violating physically feasible constraints.

This manifold is used as in classical motion planning: Once we sampled an irreducible configuration, we project this configuration down onto the constraint manifold [3]. This is schematically visualized in Fig. 3, where a random configuration on $Q$ is projected onto $Q \cap \mathcal{C}_{F M}$.

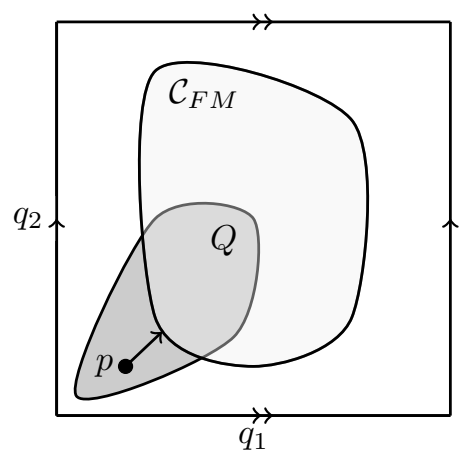

Fig. 3: Schema of a Configuration Space $\mathcal{C}$, with Constraint Manifold $\mathcal{C}_{F M} \subset \mathcal{C}$, and the irreducible manifold $Q$. A random sample $p \in Q$ is projected onto $Q \cap \mathcal{C}_{F M}$ to make it physically feasible.

\section{USE CASE: RoBOT HRP-2}

The HRP-2 Robot is represented by an open kinematic chain, whereby we have chosen $N=35$ moveable DOFs (Chest: 2, Head: 2, Left Leg: 6, Right Leg: 6, Left Arm:

\begin{tabular}{cccc} 
Joint & Fixed Value & Anatomical Name & Range \\
\hline HEAD_JOINT0 & 0 & Neck & {$[-0.52,0.79]$} \\
HEAD_JOINT1 & - & & \\
CHEST_JOINT0 & 0 & Waist & {$[-0.09,1.05]$} \\
CHEST_JOINT1 & - & & \\
RARM_JOINT & $\left(0,-\frac{\pi}{2}, 0,0,0,0\right)^{T}$ & & \\
LARM_JOINT & $\left(0, \frac{\pi}{2}, 0,0,0,0\right)^{T}$ & & \\
LLEG_JOINT0 & 0 & & {$[-2.18,0.73]$} \\
LLEG_JOINT1 & 0 & Hip & {$[-0.03,2.62]$} \\
LLEG_JOINT2 & - & Knee & \\
LLEG_JOINT3 & - & Ankle & {$[-1.31,0.73]$} \\
LLEG_JOINT4 & - & & \\
LLEG_JOINT5 & 0 & & \\
RLEG_JOINT0 & 0 & Hip & \\
RLEG_JOINT1 & 0 & Knee & \\
RLEG_JOINT2 & LLEG_JOINT2 & Ankle & \\
RLEG_JOINT3 & LLEG_JOINT3 & & \\
RLEG_JOINT4 & LLEG_JOINT4 & AlNEG & \\
RLEG_JOINT5 & 0 & Left Foot & {$[-0.5,0.5]$} \\
LSOLE_JOINT_X & - & Left Foot & {$[-3.0,3.0]$} \\
LSOLE_JOINT_Y & - & & \\
\hline
\end{tabular}

TABLE I: Variable Joints of Humanoid Robot HRP-2, and the corresponding range. All joints without a range have fixed values, which are chosen according to Theorem 6 and 7 respectively. The left foot range was chosen specifically for the wall experiments, the other ranges are given by the physical limitations of the robotic system.

6, Right Arm: 6, Free Flyer: 7). We are adding a list of assumptions, namely

1) Left Foot is oriented 90 degrees along y-axis, as depicted in Fig. 4

2) The chest has fixed $\theta_{x}$ and $\theta_{z}$ rotation

3) The head is a sphere, and so a rotation of the head along the $\mathrm{z}$-axis does not change the projected volume

Those assumptions reduce the space to $N=27$ moveable DOFs. (Chest: 1, Head: 1, Left Leg: 3, Right Leg: 6, Left Arm: 6, Right Arm: 6, Free Flyer: 4)

\section{A. Irreducible Configuration Subspace for HRP-2}

Since the chest is not rotating around $\theta_{x}, \theta_{z}$, Theorem 7 holds for all children configurations, which are covered by Theorem 5. which includes the right and the left arm. The same is true for the left leg, such that we can reduce the right leg. To summarize:

1) Left arm is reducible by chest, due to Theorem 5.7

2) Right arm is reducible by chest, due to same argument

3) Right leg is reducible by left leg, due to same argument

In this way, we assign fixed values to the left arm, right arm and right leg, chosen such that Theorem 5 applies. The values are shown in Table I and the workspace volume of the default configuration is shown in Fig. 4. It can be seen that the cross section volume of the chest covers the cross section volume of the left and right arm, and that the same applies to the right leg with respect to the left leg. This gives rise to a 7-dimensional subspace inside the configuration space. We will call it $Q_{\text {Humanoid }} \subset \mathcal{C}$.

Definition 16. Given $\mathcal{R}=H R P-2$, we define the irreducible configuration subspace of $\mathcal{R}$ as $Q_{\text {Humanoid }}=$ $\left\{q_{\text {Neck }}, q_{\text {Abdomen }}, q_{\text {Hip }}, q_{\text {Knee }}, q_{\text {Ankle }}, q_{x, \text { Left Foot }}, q_{y, \text { Left Foot }}\right\} \in \mathbb{R}^{7}$. See Table [] 

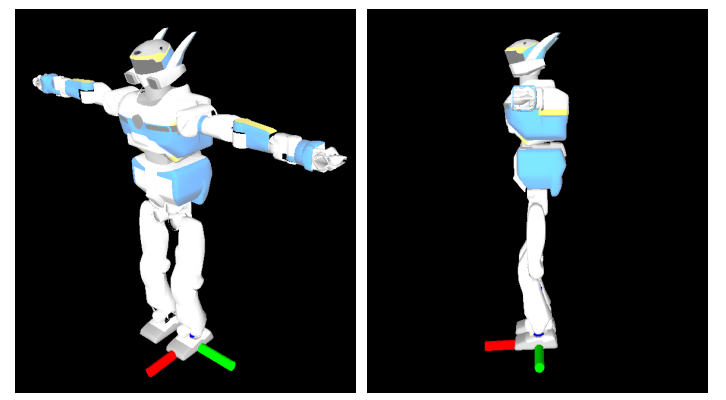

Fig. 4: Left Default Position of HRP-2 where the arms are in an irreducible configuration with respect to the chest, according to Theorem 5 Right and its view from the side, showing that the projected volume of the arms is indeed covered by the chest volume.

\section{B. Simulation Results}

To test our theoretical results, we have chosen a motion planning problem, where the robot HRP-2 has to move through a wall, as shown in Fig. 5. Due to the wall constraint, a solver has to find a narrow passage in the configuration space to solve the problem. In the classical 35-dof setting, this problem has been solved by adding 12 waypoints to guide the planning algorithm [3]. We consider here the 7-dof setting without waypoints, by using the irreducible configuration subspace. Since the initial position and the goal position are not irreducible, we project them onto the irreducible configuration subspace.

Our algorithm uses a standard RRT implementation [13], plans from the scratch without waypoints, and is using the irreducible configuration subspace for sampling. The results of 10 runs are reported in Table II Since the passage is narrow, RRT can take a long time to converge, for our experiment, it took between 44 minutes up to 43 hours. Even so RRT is considered state-of-the-art in motion planning [13], the results highlight, that it might not be the preferred method for problems involving narrow passages in configuration space.

A video is available showing the resulting trajectory of the first run:

\section{http://goo.gl/IdTJuv}

Since this paper is concerned with a feasibility study, the resulting motion will be non-optimal, assumes infinitesimal small footsteps and might appear unnatural to a human observer. However, having a first feasible trajectory is a prerequisite for fast convergence of local planning algorithms like CHOMP [15] or AICO [16].

TABLE II: Simulation results for the wall motion planning problem. Each Instance is a basic RRT without prior information, planning in $Q_{\text {Humanoid }}$.

\begin{tabular}{cc}
\hline Planning Instance & Planning Time \\
\hline 0 & $3 \mathrm{~h} 37 \mathrm{~m}$ \\
1 & $6 \mathrm{~h} 07 \mathrm{~m}$ \\
2 & $3 \mathrm{~h} 55 \mathrm{~m}$ \\
3 & $9 \mathrm{~h} 34 \mathrm{~m}$ \\
4 & $6 \mathrm{~h} 37 \mathrm{~m}$ \\
5 & $\mathbf{0 h 4 4 m}$ \\
6 & $16 \mathrm{~h} 19 \mathrm{~m}$ \\
7 & $2 \mathrm{~h} 45 \mathrm{~m}$ \\
8 & $43 \mathrm{~h} 57 \mathrm{~m}$ \\
9 & $2 \mathrm{~h} 03 \mathrm{~m}$ \\
\hline
\end{tabular}
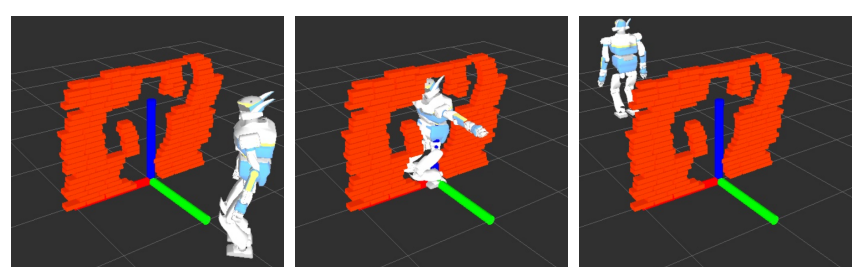

Fig. 5: Wall Motion Planning Problem. Left initial configuration Middle one irreducible configuration on the final trajectory found by an RRT on the irreducible subspace Right goal configuration.

\section{CONCLUSION}

We defined the irreducible property for configuration space trajectories, trajectories which have a minimal swept volume, such that there is no other trajectory with a smaller swept volume. Our main result is given by Theorem 2 , which states that finding no feasible trajectory in the space of irreducible trajectories implies that there is no feasible trajectory in the space of all configuration space trajectories, i.e. that motion planning is complete w.r.t. irreducible trajectories.

In practice, we approximated the swept volume by its cross sections, defined the irreducible property on those cross sections, and used those results under mild assumptions to define a low dimensional subspace for the humanoid robot HRP-2. This subspace allowed us to find a feasible trajectory in a highly constraint environment, where previous planners could only find a solution by using waypoints [3].

Since this approach will find a feasible trajectory only in the space of irreducible trajectories, the obtained motions are possibly not natural or unaesthetic from the view of an external observer. However, once we found such a feasible trajectory, we can make use of advanced control algorithms or local motion planning techniques, in order to deform our motion locally.

Future research will focus on more efficient planning algorithms for irreducible trajectories and on conditions for completeness of the cross section operator.

\section{Proofs}

Proof of Theorem 1. Let $s=\mathcal{S} \mathcal{V}(\tau)$ and $s^{\prime}=\mathcal{S V}\left(\tau^{\prime}\right) . s$ is feasible if $s \cap \mathbf{E}=\emptyset$. We proceed by direct proof:

(1) Let $s$ be infeasible, then $\exists v \in s$, such that $v \cap \mathbf{E}=v$. Since $s \subset s^{\prime}$, we have that $v \in s^{\prime}$. Since $v$ exists, we can conclude that at least $s^{\prime} \cap \mathcal{E}>v$, which makes $s^{\prime}$ infeasible. (2) $\tau^{\prime}$ being feasible means $s^{\prime} \cap \mathbf{E}=\emptyset$. Since $s \subset s^{\prime}$, it follows from elementary set theory that $s \cap \mathbf{E}=\emptyset$, which proofs that $\tau$ is feasible.

Proof of Lemma 1. Let $\mu$ be the lebesque measure on the workspace $\mathcal{W}$. First, let us see that if $\mathcal{S} \mathcal{V}\left(\tau_{1}\right) \subset \mathcal{S} \mathcal{V}\left(\tau_{0}\right)$, then $\mu\left(\mathcal{S V}\left(\tau_{1}\right)\right)<\mu\left(\mathcal{S} \mathcal{V}\left(\tau_{0}\right)\right)$.

Now, by definition, if $\tau_{0} \notin \mathcal{I}$, then $\exists \tau_{1} \in \mathcal{F}$, such that $\mathcal{S} \mathcal{V}\left(\tau_{1}\right) \subset \mathcal{S} \mathcal{V}\left(\tau_{0}\right)$. Then either $\tau_{1} \in \mathcal{I}$, and we are done. Or $\tau_{1} \notin \mathcal{I}$, and by definition, $\exists \tau_{2} \in \mathcal{F}$, such that $\mathcal{S} \mathcal{V}\left(\tau_{2}\right) \subset \mathcal{S} \mathcal{V}\left(\tau_{1}\right)$. Let us assume that there is no trajectory $\tau_{i} \in \mathcal{I}$, such that we obtain an infinite sequence $\Pi=\left\{\tau_{0}, \tau_{1}, \tau_{2}, \cdots\right\}$ of reducible trajectories $\tau_{i} \in \mathcal{F}$, such that $\forall \tau_{i} \in \Pi: \mathcal{S} \mathcal{V}\left(\tau_{i+1}\right) \subset \mathcal{S} \mathcal{V}\left(\tau_{i}\right)$. Since we have $\forall \tau_{i} \in \Pi$ : 
$\mu\left(\mathcal{S} \mathcal{V}\left(\tau_{i+1}\right)\right)<\mu\left(\mathcal{S} \mathcal{V}\left(\tau_{i}\right)\right)$ and $\mu(\mathcal{S V}(\tau))>0$, the sequence is strictly monotonically decreasing and bounded, and will therefore converge to its maximum lower bound, which we call $C$, i.e. $\lim _{n \rightarrow \infty} \mu\left(\mathcal{S V}\left(\tau_{i}\right)\right)=C$. Consequently, since the maximum lower bound is obtained, there cannot exists another trajectory $\tau^{\prime}$, such that $\mu\left(\mathcal{S} \mathcal{V}\left(\tau^{\prime}\right)\right)<C$. By definition, the sequence is converged in $\mathcal{I}$, and therefore we conclude that every element $\tau \in \mathcal{F} \backslash \mathcal{I}$ is reducible by $\tau^{\prime} \in \mathcal{I}$.

Proof of Theorem 2. Let us assume that $\exists \tau \in \mathcal{F}$, with $\tau$ being feasible, and that $\forall \tau^{\prime} \in \mathcal{I}: \tau^{\prime}$ is not feasible. Since $\tau$ is feasible, it follows that $\tau \notin \mathcal{I}$. Then by definition there has to be a $\tau^{\prime \prime} \in \mathcal{F}$ such that $\mathcal{S} \mathcal{V}\left(\tau^{\prime \prime}\right) \subset \mathcal{S} \mathcal{V}(\tau)$. Then $\tau^{\prime \prime}$ is feasible by Theorem 1 . Further, either we have that $\tau^{\prime \prime} \in \mathcal{I}$. Then we have a contradiction. Or we have $\tau^{\prime \prime} \notin \mathcal{I}$, which means that we can still find another $\tau^{\prime \prime \prime} \in \mathcal{F}$ reducing $\tau^{\prime \prime}$. By Lemma 1. we know that such a sequence can be reduced by a $\tilde{\tau} \in \mathcal{I}$. So we reach a contradiction, too.

Proof of Corollary 1. By definition, motion planning is complete, if we can find a solution (a trajectory), if one exist. By Theorem 2, we know that if we cannot find a solution in $\mathcal{I}$, then there is no solution in $\mathcal{F}$. Conversely, if there is a solution in $\mathcal{F}$, then by Theorem 1 , there exists a solution in $\mathcal{I}$.

Proof of Theorem 3. 1) It follows directly from $V(q) \subset V\left(q^{\prime}\right)$, i.e. if $q$ is infeasible, then $\exists v \in V(q)$, such that $v$ intersects the environment, i.e. $v \cap \mathbf{E} \neq \emptyset$. Because we have $v \in V(q) \Rightarrow v \in V\left(q^{\prime}\right)$, we have that $\exists v \in V\left(q^{\prime}\right)$, such that it intersects the environment, and makes $q^{\prime}$ therefore infeasible.

2) Similar, if $q^{\prime}$ is feasible, then $\forall v \in V\left(q^{\prime}\right)$ we have $v \cap \mathbf{E}=\emptyset$. Since $v \in V(q) \Rightarrow v \in V\left(q^{\prime}\right)$, we have that $v \in V(q) \Rightarrow v \cap \mathbf{E}=\emptyset$, and so $q$ is feasible.

Proof of Theorem 4. Let us assume that $\forall q \in Q$ : $q$ is infeasible. Let us assume that $\exists q \in \mathcal{C}, q \notin Q$, and that $q$ is feasible. Since $q \notin Q$, we know that $\exists q^{\prime}$, which reduces $q$. Then by Theorem $3 q^{\prime}$ is feasible, too. Now, there are two cases: Either $q^{\prime} \in Q$, then we have a contradiction. Or $q^{\prime} \notin Q$, then we can find another $q^{\prime \prime}$, which reduces $q^{\prime}$, meaning that we can reduce $q$ until we reach a contradiction, according to a similar argument as made in Lemma 1

Proof of Lemma 2.

$$
\begin{aligned}
& (\Rightarrow) \mathbf{T}(V(p)) \subset \mathbf{T}(V(q)) \\
& \quad \Rightarrow V(p)+\mathbf{T} \subset V(q)+\mathbf{T} \Rightarrow V(p) \subset V(q) \\
& (\Leftarrow) V(p) \subset V(q) \\
& \quad \Rightarrow V(p)+\mathbf{T} \subset V(q)+\mathbf{T} \Rightarrow \mathbf{T}(V(p)) \subset \mathbf{T}(V(q))
\end{aligned}
$$

Proof of Lemma 3. Since $V(p) \subset V(q)$, we have $\forall v \in$ $V(p) \rightarrow \exists v^{\prime} \in V(q)$, such that $v=v^{\prime}$. Rotation is equivalent to applying $\mathbf{R}_{Y}$ onto both sites to get $\mathbf{R}_{\mathrm{Y}} v=$ $\mathbf{R}_{\mathrm{Y}} v^{\prime}$. Now, let $V(p)=\left\{v_{1}, v_{2}, \cdots\right\}$. Applying $\mathbf{R}_{\mathrm{Y}}$ gives $\mathbf{R}_{\mathrm{Y}}(V(p))=\left\{\mathbf{R}_{\mathrm{Y}} v_{1}, \mathbf{R}_{\mathrm{Y}} v_{2}, \cdots\right\}$. Since $v_{i}=v_{i}^{\prime} \in V(q)$, $\mathbf{R}_{\mathrm{Y}} v_{i}=\mathbf{R}_{\mathrm{Y}} v_{i}^{\prime}$, we conclude that $\mathbf{R}_{\mathrm{Y}}(V(p)) \subset \mathbf{R}_{\mathrm{Y}}(V(q))$. And vice versa.

Proof of Theorem 5. Assume that $\exists \tilde{c} \in \mathcal{C}_{c}\left(N_{j}\right)$, such that $V(\tilde{c} \cup p) \subset V\left(c^{\prime} \cup p\right)$, i.e. $c^{\prime} \cup p$ is reducible by $\tilde{c} \cup p$. We know that $V\left(c^{\prime} \cup p\right)=V\left(c^{\prime}\right) \cup V(p)$ and that $V\left(c^{\prime}\right) \subset V(p)$. Therefore, $V\left(c^{\prime}\right) \cup V(p) \subset V(p)$. Therefore, $V(\tilde{c} \cup p) \subset$ $V\left(c^{\prime} \cup p\right) \subset V(p)$. However, clearly we have $V(\tilde{c}) \cup V(p) \supset$ $V(p)$, which contradicts our assumption, and we follow that there does not exist such an element.

Proof of Theorem 6. Follows by applying Lemma 2 onto $V\left(c^{\prime} \cup p\right) \subset V(c \cup p)$

Proof of Theorem 7. Follows by applying Lemma 3 onto $V\left(c^{\prime} \cup p\right) \subset V(c \cup p)$

\section{REFERENCES}

[1] J. F. Canny, The complexity of robot motion planning MIT press, 1988.

[2] A. Escande, A. Kheddar, and S. Miossec, 'Planning contact points for humanoid robots" Robotics and Autonomous Systems, vol. 61, no. 5, pp. $428-442,2013$.

[3] A. El Khoury, F. Lamiraux, and M. Taix, 'Optimal Motion Planning for Humanoid Robots." in International Conference on Robotics and Automation, 2013.

[4] K. Hauser, Motion planning for legged and humanoid robots $\mathrm{PhD}$ thesis, Departement of Computer Science, Stanford University, 2008.

[5] O. Brock and L. E. Kavraki, 'Decomposition-based Motion Planning: A Framework for Real-time Motion Planning in High-dimensional Configuration Spaces" in International Conference on Robotics and Automation, 2001.

[6] J. Pan, S. Chitta, and D. Manocha, 'Faster Sample-based Motion Planning using Instance-based Learning" in Algorithmic Foundations of Robotics X, Springer, 2013.

[7] M. Phillips, B. J. Cohen, S. Chitta, and M. Likhachev, 'E-Graphs: Bootstrapping Planning with Experience Graphs " in Robotics: Science and Systems, 2012.

[8] N. Jetchev and M. Toussaint, "Fast motion planning from experi-" ence: trajectory prediction for speeding up movement generation" Autonomous Robots, 2013.

[9] K. Hauser, T. Bretl, K. Harada, and J.-C. Latombe, 'Using motion primitives in probabilistic sample-based planning for humanoid robots" in Algorithmic foundation of robotics VII, pp. 507-522, Springer, 2008

[10] D. Berenson, P. Abbeel, and K. Goldberg, 'A robot path planning framework that learns from experience" in International Conference on Robotics and Automation, 2012.

[11] A. Majumdar and R. Tedrake, 'Robust online motion planning with regions of finite time invariance" in Algorithmic Foundations of Robotics X, Springer, 2013.

[12] A. J. Ijspeert, J. Nakanishi, H. Hoffmann, P. Pastor, and S. Schaal, 'Dynamical Movement Primitives: Learning Attractor Models for Motor Behaviors" Neural computation, 2013.

[13] S. M. LaValle, Planning Algorithms Cambridge University Press, 2006.

[14] J. C. Himmelstein, E. Ferre, and J.-P. Laumond, "Swept volume approximation of polygon soups" IEEE Transactions on Automation Science and Engineering, 2010.

[15] M. Zucker, N. Ratliff, A. Dragan, M. Pivtoraiko, M. Klingensmith, C. Dellin, J. A. D. Bagnell, and S. Srinivasa, 'CHOMP: Covariant Hamiltonian Optimization for Motion Planning," International Journal of Robotics Research, May 2013.

[16] M. Toussaint, 'Robot trajectory optimization using approximate inference" in International Conference on Machine Learning, 2009. 